\title{
Sacrificing a Little Coverage Can Substantially Increase Network Lifetime ${ }^{1}$
}

\author{
Limin Wang Sandeep S. Kulkarni \\ Software Engineering and Network Systems Laboratory \\ Department of Computer Science and Engineering \\ Michigan State University \\ East Lansing MI 48824 USA \\ Email: \{wanglim1, sandeep\}@cse.msu.edu
}

\begin{abstract}
We present a simple, local protocol, pCover, which provides partial (but high) coverage in sensor networks. Through pCover, we demonstrate that it is feasible to maintain a high coverage $(\sim 90 \%)$ while significantly increasing coverage duration when compared with protocols that provide full coverage. In particular, we show that we are able to maintain 94\% coverage for a duration that is 2.3-7 times the duration for which existing protocols maintain full coverage. Through simulations, we show that our protocol provides load balancing, i.e., the desired level of coverage is maintained (almost) until the point where all sensors deplete their batteries. We also show that $\mathrm{pCover}$ handles failure of sensors, different coverage areas, different node densities, and different topologies, and can be used for dynamically changing the level of coverage.
\end{abstract}

Keywords: sensor networks, node scheduling, partial coverage, lifetime, power conservation

1 This work was partially sponsored by NSF CAREER CCR-0092724, DARPA Grant OSURS01-C-1901, ONR Grant N00014-01-1-0744, NSF equipment grant EIA-0130724, and a grant from Michigan State University. 


\section{Introduction}

A surveillance sensor network needs to operate unattended for a long time, usually from several weeks to several months. However, sensor nodes are usually battery-powered, and, hence, can only operate continuously for a few days. Therefore, energy conservation operations are critical for extending network lifetime. In this paper, we consider the approach where sensor nodes are over-deployed in a given area, only a subset of the nodes are in active mode to maintain a certain degree of sensing coverage (based on the desired system functionality), and the remaining ones are put in sleeping mode.

The work on sensing coverage can be broadly classified in terms of those that provide full coverage (single coverage or multiple coverage $[9,11,12])$ and those that provide partial coverage. In full coverage, every point in the network is covered by at least one sensor. While such coverage is desirable in sensitive environments such as military surveillance, it requires a large number of sensors to be awake. In partial coverage, by contrast, only a subset of points in the sensor network are covered and, hence, the number of sensors that need to be awake is reduced.

In this paper, we focus on high partial coverage ( $~ 90 \%$ coverage), that we expect to combine benefits of full coverage (better surveillance) and partial coverage (longer lifetime). With high partial coverage, at any time, the intruder is likely to be detected with a high probability. Moreover, because the active nodes change over time, a stationary intruder will be detected with certainty in a short period. Finally, a moving intruder will also be detected within short distance. Such high partial coverage is desirable in many scenarios. For example, in a forest fire detection system, high partial coverage will ensure that most fires are detected immediately and all fires are detected within a short duration. Likewise, high partial coverage could also be used in applications such as ExScal [6], an application for intruder detection, classification and tracking.

We show that providing high (partial) coverage can significantly increase the lifetime of a given sensor network compared to the cases that provide full coverage. To illustrate this, we present a simple local protocol, $p$ Cover, that provides partial coverage. $p$ Cover is not dependent on global properties such as time synchronization. The degree of coverage is controllable locally. This is needed when network characteristics change. For example, sensing range changes due to environmental changes (grass, temperature, rain, etc.) or reduced battery level. In such case, we should be able to dynamically tune the degree of coverage by simply sending a parameter to all the sensor nodes in the network. In a network with uniform deployment, the desired degree of coverage is maintained (almost) to the point where all sensor nodes fail. As illustrated in Section 5.6, along with a local algorithm, this feature can also be used to provide gradually decreasing coverage.

Our protocol provides a simple and practical solution for a partially covered sensor network. It can be used to provide full 
coverage as well. When used to provide guaranteed $100 \%$ coverage, pCover performs similarly as the state-of-art protocol that provides full coverage [23]. We also illustrate that $p$ Cover performs well at different topologies, different network densities and different coverage areas, and is able to tolerate failure of sensors gracefully.

Contributions of the paper. We present a protocol, pCover, which maintains a certain degree of sensing coverage through sleep-awake scheduling of sensor nodes. Through simulation of $p$ Cover, we analyze the tradeoff between sensing coverage and network lifetime, evaluate it in context of different network topologies, different node densities, different coverage areas, and sensor failures. We show that sacrificing a little sensing coverage can substantially increase the lifetime of the network, compared to protocols that provide full coverage (e.g., [19,23]). In particular, in a random uniform deployment, our protocol maintains $94 \%$ coverage for a duration that is 2.3 times (respectively, 7 times) the duration for which the protocol in [23] (respectively, [19]) maintains full coverage. If the desired coverage is $91 \%$, the duration that this coverage is maintained is increased to 2.6 times (respectively, 7.9 times) the duration that [23] (respectively, [19]) maintains full coverage. If the desired coverage is $84 \%$, the duration that this coverage is maintained is 3.4 times (respectively, 10.3 times) the duration that [23] (respectively, [19]) maintains full coverage. And we show that $p$ Cover maintains load balancing, i.e., the degree of sensing coverage is maintained at desired level (almost) until the point where all sensor nodes deplete their batteries. We also show the battery levels of sensors over time to illustrate that sensors lose power uniformly. In addition, we identify the lower bound of the partial coverage that $p$ Cover can provide.

Organization of the paper. In Section 2, we identify the system model and assumptions of the partial coverage problem. In Section 3, we present our partial coverage protocol, pCover. In Section 4, we analyze the effect of the configurable parameters in our protocol. In Section 5, we show the tradeoff between coverage and network lifetime, and evaluate the performance of $p$ Cover. In Section 6, we compare the duration that $p$ Cover maintains high partial coverage and the duration that $p$ Cover maintains full coverage. We also compare $p$ Cover with other state-of-art protocols $[19,23]$ that provide guaranteed full coverage. In Section 7, we propose an extension to improve the quality of surveillance. We survey related work in Section 8, and conclude in Section 9.

\section{System Model and Assumptions}

We consider a network with stationary sensor nodes. Each node knows its location (through some localization service) and its sensing range. The sensing ranges of sensor nodes do not necessarily need to be the same. The different sensing 
ranges can be caused by the hardware (antenna, radio unit, etc.), the remaining power levels (which can change during nodes' lifetime), or the territory conditions.

We define the neighbor set of a node $i$ as the set of nodes whose sensing areas intersect with the sensing area of node $i$. For simplicity, we assume that the sensing area of a node $i$ is a circle with radius $r_{i}$ centered at the location of the node. Hence, the neighbor set of node $i$ is

$$
N(i)=\left\{j \in R \mid d(i, j) \leq\left(r_{i}+r_{j}\right), j \neq i\right\}
$$

where $R$ is the entire set of sensor nodes in the target region, $d(i, j)$ is the distance between node $i$ and node $j$.

A simple case is where all nodes have the same sensing range $r$. In this case, the neighbor set of a node $i$ is

$$
N(i)=\{j \in R \mid d(i, j) \leq 2 r, j \neq i\}
$$

We assume that the radio communication range is at least the distance to the farthest neighbor (i.e., no less than $2 r$, in the case of equal sensing range). This assumption holds for most existing sensor products, such as [5].

The partial coverage problem we address in this paper can be described as follows. The sensor nodes are deployed with redundant density. Sensor nodes self-organize to achieve a desired degree of coverage. This degree of coverage is maintained until some of the nodes have drained out power such that there are not enough nodes left to satisfy the coverage requirement.

\section{3 pCover: Protocol Description}

In this section, we present our partial coverage protocol, pCover. In $p$ Cover, a sensor node is either in working mode or sleeping mode. In Section 3.1, we present the local rule that a sensor node follows to determine whether it should go to sleep or stay awake. In Section 3.2, we describe state transition and how this local rule is used.

\subsection{Sleep Eligibility Rule}

We first define the terms we are going to use when we describe the local rule and the protocol.

Global coverage is the percentage of the target area that is covered by the working nodes. This is also called the degree of coverage.

Local coverage ${ }^{2}$ of a node is the percentage of the node's sensing area that is covered by its awake neighbors.

\footnotetext{
${ }^{2}$ Note that the defi nition does not assume geometry of a node's sensing area. Hence, $p$ Cover can be applied to the case where sensing area is not a circle as well.
} 
Contribution of a node is the percentage of the node's sensing area that is not covered by its awake neighbors, i.e., Contribution $=1-$ LocalCoverage.

The basic idea of the sleep eligibility rule is that: a sensor node should turn itself off if and only if its local coverage (respectively, contribution) is higher (respectively, lower) than a certain threshold, SleepThreshold (respectively, 1-SleepThreshold).

To facilitate calculation, we imagine that the target area is covered by a virtual grid (Figure 1), where the size of a grid point is significantly smaller than the sensing area of a sensor node. In Figure 1, when A calculates its eligibility to sleep, it counts the number of grid points, say TotalGrid, that are within its sensing range. It also calculates the set of grid points, say CoveredGrid, that are covered by its awake neighbors. Thus, A can compute its local coverage (CoveredGrid/TotalGrid) and contribution, and decide if it should go to sleep or stay awake.

\subsection{State Transition}

In this section, we describe the state transition in pCover (Figure 2). Each node is in one of 4 states: probing, awake, readyoff, and sleep. Nodes switch among these states until they run out of power. Each node keeps a neighbor table, which maintains one record (ID, location, sensing range, status) for each neighbor.

A node in probing state probes the environment, evaluates the sleep eligibility rule, and decides if it should start working or go to sleep. A node in awake state is a working node. It monitors the area within its sensing range, and contributes to global coverage. A node in readyoff state is one that wants to sleep but cannot do that until its local coverage is higher than a certain threshold. It is also a working node, and contributes to global sensing coverage. It announces its intention to sleep so that its neighbors do not count it when they calculate their sleep eligibility, and, hence, are more likely to stay awake. A node in sleep state only keeps a timer on, and turns off all the sensing, communication, and computation devices, in order to save energy. Now we describe the actions a sensor node performs in these states.

Actions in Probing State. In the initial stage, all the nodes are in probing state. Each node waits for a random back-off time, and then broadcasts a probing message. The probing message contains the node's ID, location and sensing range. It is used to collect awake neighbors' information. Whenever a neighbor node receives this probing message, if it is in awake state, it sends back a probing reply message, which also contains its ID, location and sensing range. Nodes update their neighbor tables based on the neighbor information they have collected, compute the sleep eligibility rule, and decide if they 
should go to sleep or stay awake.

When a node in probing state computes the sleep eligibility rule, it applies a SleepThreshold, which we call on-threshold. A probing node starts working if it finds that its local coverage is lower than on-threshold.

The random back-off time is used for two purposes. First, the probing messages are sent at different time, so that they are unlikely to collide. Second, nodes should decide their states in sequential order. For example, consider node A and node B in Figure 1, if A and B try to decide their states at the same time, since neither of them is in awake state at the moment (both are in probing state), they are not counted for each other's local coverage. As a result, the redundancy increases.

If a node decides to stay awake, it broadcasts an "AnnounceAwake" message, and switches to awake state. On the other hand, if a node decides to go to sleep, it turns off its radio and sensing units, and goes to sleep state.

Actions in Awake State. A node in awake state actively monitors the area within its sensing range. It remains in awake state for $T_{w}$ period of time ( $T_{w}$ could be chosen deterministically or randomly), then changes its state to readyoff, and broadcasts a "ReadyToOff" message. By sending a "ReadyToOff" message, the node indicates a desire to turn off.

Actions in Readyoff State. Intuitively, readyoff state is a reception only state, i.e., a node in readyoff state does not respond to any probing messages. Therefore, a readyoff node appears to its neighbors as a sleeping node. However, a readyoff node is in fact a working node, and provides sensing coverage. A node in readyoff state does not turn itself off until enough of its neighbors are awake to take over its sensing area. However, since it does not reply to probing messages, its neighbors do not count it when they compute their sleep eligibility. Also, a readyoff node watches for "AnnounceAwake" and "ReadyToOff" messages from its neighbors, and recomputes its sleep eligibility whenever it notices an increase in its local coverage. The SleepThreshold we apply here is off-threshold. If a readyoff node finds that its local coverage is higher than off-threshold, it will go to sleep state.

We also include an optional transition from readyoff state to awake state. A node that is in readyoff state for a long duration can switch to awake state and send an AnnounceAwake message. This transition allows one to deal with the case where a lot of nodes are in readyoff state although none of them can go to sleep state. Through simulations, we however found that a transition from readyoff state to awake state occurs only when there are no other alive nodes that can enable the node in readyoff state to sleep. In other words, in such a case, a node will continue to switch between awake state and readyoff state, thereby continually contributing to sensing coverage. However, in a real network, if the state of a node is corrupted for some reason, this transition will allow one to recover from the state where all nodes (large number of nodes) are erroneously in readyoff state. 
Actions in Sleep State. A node in sleep state wakes up every $T_{s}$ period of time. When it wakes up, it performs two tasks. First, it senses its surrounding area (within its sensing range) to see if there is any interesting event. This guarantees that any event that lasts longer than $T_{s}$ will always be detected as long as there are alive sensor nodes in the area where the event occurs. Second, it changes its state to probing (and proceeds to execute actions in probing state).

We note that a node refreshes its neighbor table periodically. Therefore it provides a certain level of fault tolerance. We assume that when a node fails, it simply stops working and does not send or receive any messages. If a node that was in awake state fails, its neighbors will be able to detect it within $T_{s}$, at the time when they wake up, probe the neighborhood and get the updated neighbor information.

\section{Analysis of On-threshold and Off-threshold}

Since the goal of this work is to identify the tradeoff between full coverage and high partial coverage, we use the setting in [23], where the authors have proposed a protocol that is able to provide full coverage. In particular, the sensor nodes are deployed in a $160 \mathrm{~m} \times 160 \mathrm{~m}$ field (called deployment area). The target area is the $140 \mathrm{~m} \times 140 \mathrm{~m}$ square in the center of the field. Moreover, when we calculate the number of nodes, we only consider those in the central $100 \mathrm{~m} \times 100 \mathrm{~m}$ area (called central area). The coverage area of each sensor is a circle of radius $r=10 \mathrm{~m}$.

In all the experiments, we set $T_{s}$ to be 2 minutes, and $T_{w}$ to be 10 minutes. We assume the lifetime of a sensor node is 1000 minutes, i.e., it is able to continuously work for 1000 minutes. We consider a network as "dead" when the global coverage of the network is less than a certain threshold even if all the alive nodes are working. We define the lifetime of a sensor network as the duration from the beginning of deployment until the network is dead. We use $50 \%$ as the threshold in our experiments. However, we found that the result is almost the same if a different threshold is used, as all the nodes ran out of power within a short period of time at the end of the simulation.(The network lifetime could also be defined not only in terms of coverage but also in terms of additional requirements such as connectivity. In this context, we note that in our simulations, the network remains connected for scenarios where high coverage is provided. Moreover, if the communication range is greater than $4 *$ sensing range (which is true for sensor platforms such as Mica/Mica2, etc.), then connectivity can be theoretically shown in the context of $p$ Cover for geometric topologies such as grids with sufficient density. However, this issue is outside the scope of this paper.)

We analyze off-threshold and on-threshold in Sections 4.1 and 4.2 respectively. The simulations are conducted on a grid 
topology. For simplicity, we ignore communication cost. In Section 5.1, we show that this is reasonable in that communication cost is very small and affects network lifetime by less than $1 \%$.

In our simulation, we also assume that there is no message collision or loss. ( The same assumption was made in [23].) In pCover, we use a random back-off scheme to avoid message collisions. However, message collision is still possible. In case that the probing or probing reply messages collide, we can extend our protocol by allowing a sensor node to send a probing message twice. In the second probing message, the node includes the IDs of the neighbors it has already heard from, so that these neighbors will not send reply messages repeatedly.

\subsection{Varying Off-threshold}

We set the distance between two neighbor nodes to be $7 \mathrm{~m}$, thus 196 sensor nodes (in a $14 \times 14$ grid) fall in the central $100 \mathrm{~m} \times 100 \mathrm{~m}$ area (node density is 1.96 nodes/ $\mathrm{r}^{*} \mathrm{r}$ ). We fix the on-threshold at 0.9 , and vary the off-threshold from 0.1 to 0.9 . The samples of global coverage are obtained periodically. When computing the global coverage, we count the percentage of grid points in the target area covered by working (awake or readyoff) nodes. In Figure 3, we show the global coverage and lifetime of the network under different off-thresholds.

From Figure 3 (a), we can see that, when the on-threshold is set to 0.9 , global coverage is maintained at around $98 \%$. The curves representing the global coverage when different off-thresholds are used, overlap each other and appear indistinguishable. In Figure 3 (b) and (c), we show the average global coverage and lifetime of the network under different off-thresholds. We can see that, when off-threshold varies from 0.1 to 0.9 , the average global coverage and network lifetime varies within a short range (i.e., the global coverage varies from 0.976 to 0.982 , and the lifetime of the network varies from 3315 to 3182 minutes). Varying off-thresholds does not significantly affect network lifetime or global coverage.

From Figure 3 (a), we also note that under all off-thresholds, the global coverage is well maintained until the point of 3050 minutes, after which, the global coverage drops suddenly, and the network dies in a short period. To further illustrate the situation, we show the number of alive nodes over time, under different off-thresholds, in Figure 4. We found that the curves shown in Figure 4 are similar to those in Figure 3 (a). All the nodes (196 in total) are alive until the point of 2500 minutes, then within a short period, all of them run out of power. This indicates that our algorithm maintains a balanced energy consumption.

We also simulate our algorithm when on-threshold is changed to a lower value, 0.6 , and other settings remain the same. We still vary off-threshold from 0.1 to 0.9. We show the global coverage over time in Figure 5 (a), and the average global 
coverage and network lifetime under different off-thresholds in Figure 5 (b) and (c). Similar to the situation where we use a higher on-threshold 0.9 (Figure 3), when off-threshold increases from 0.1 to 0.7 , the change in off-threshold has little impact on the global coverage and network lifetime. As shown in Figure 5, the global coverage varies within a short range from 0.899 to 0.911 (Figure 5 (a) and (b)), and the network lifetime changes from 5200 and 5014 minutes (Figure 5 (c)). However, when the off-threshold is high (higher than 0.7), the global coverage increases relatively fast with the increment of the offthreshold. As shown in Figure 5, when the off-threshold increases from 0.7 to 0.9 , the global coverage increases from 0.911 to 0.974 , and the network lifetime decreases from 5014 to 3792 minutes.

From these experiments, we conclude that off-threshold does not have much impact on global coverage or network lifetime, as long as it is not set to too high (e.g., higher than 0.7). In particular, if the off-threshold is within the range of 0.1-0.6, global coverage and lifetime of a given network are mainly decided by the on-threshold.

\subsection{Varying On-threshold}

Based on the observation from Section 4.1, the off-threshold does not affect global coverage and network lifetime much as long as it is not greater than 0.7 . Hence, in this section, we fix off-threshold at 0.6 , and study the effect of on-threshold on network lifetime and global coverage when it varies from 0.3 to 0.9 . We set the inter-node distance to $7 \mathrm{~m}$. This makes the node density to be 1.96 nodes/r*r. In Figure 6, we show global coverage and lifetime of the network. We find that global coverage (respectively, network lifetime) increases (respectively, decreases) linearly with the increment of on-threshold. This indicates that on-threshold can be used to locally control global coverage and network lifetime.

We note that global coverage varies within a relatively short range (14\%, from $84 \%$ to $98 \%$ ), while network lifetime varies significantly (from 3300 minutes to 6400 minutes, almost doubles). From Figure 6 (a), we also found that under all on-thresholds, the global coverage is well maintained until one point, after which, the global coverage drops suddenly, and the network dies in a short period. This indicates that our algorithm maintains a balanced energy consumption as all sensor nodes run out of power at around the same time.

\section{Performance of $p$ Cover}

In Sections 5.1 and 5.2, we study the lifetime-coverage tradeoff provided by $p$ Cover under two distribution patterns: deterministic distribution and random distribution. In the first case, sensors nodes are deployed in a grid topology. In the 
second case, nodes are deployed in a random manner. In Section 5.3, we investigate the case where the coverage area is not a circle, in particular, is a sector. In Section 5.4, we analyze the quality of surveillance. In Section 5.5, we demonstrate the uniform energy consumption of sensors at different times. In Section 5.6, we show that by changing the parameters, it is possible to control the level of coverage dynamically. By default, we assume that the coverage area of a sensor is a circle centered at the sensor. In Section 5.7, we study the behavior of pCover in the presence of sensor failures. In Section 5.8, we identify the limits for coverage that $p$ Cover, as a "local" protocol, can provide.

\subsection{Global Coverage and Network Lifetime in Networks with Deterministic Topology}

We experiment on different node densities. In Figure 7 (a), we show the relationship between global coverage and onthreshold. We can see that, global coverage linearly increases with the increment of on-threshold. Moreover, the curves that represent global coverage under different node densities are almost overlapping, with the exception of the node density being 1 node/r*r. In the case when the node density is as low as 1 node/r*r, the network is too sparse to meet the coverage requirement. It shows that global coverage is largely decided by on-threshold. Furthermore, when node density is reasonably high (e.g., not lower than 2 nodes/r*r), global coverage is independent of node density. In Figure 7 (b), we show that network lifetime decreases linearly when on-threshold increases. The space between the lines is even. Thus, if we fix the on-threshold, the lifetime of the network is proportional to the node density.

In Figure 7 (c), we show the tradeoff between global coverage and network lifetime, under different node densities. As we have already noticed from Figure 7 (a) and (b), when node density is as low as 1 node/r*r, the highest global coverage of a network that can be achieved is around $90 \%$. When node density is higher, the limitation on the highest achievable global coverage no longer exists. Depending on the node density, decreasing the global coverage by $1 \%$ increases the network lifetime by 210 minutes (at 1.96 nodes $/ \mathrm{r} * \mathrm{r}$ ) to 450 minutes (at 4 nodes $/ \mathrm{r} * \mathrm{r}$ ).

We now set on-threshold to 0.7 , off-threshold to 0.6 , and vary the density of the network from 1 node/r*r to 4 nodes/r*r. We show the change of global coverage over time for different node densities in Figure 8 (a). We find that global coverage is maintained at the same level (around 94\%) under all densities, except the case when node density is 1 node/r*r (also shown in Figure 8 (b)).

In Figure 8 (b), we show that the global coverage is decided by the sleep thresholds (mainly on-threshold), independent of the density of the network. This is desirable, since it enables sensor nodes to control the global coverage locally by tuning the sleep threshold (on-threshold) without knowing the density of the network. The lifetime of the network is linearly increasing 
with the increment of node density. This indicates that if we want to double the lifetime of the network without changing the global coverage, we could simply double node density in the target area.

Remarks. In our simulation, we did not consider communication overhead, as the energy consumed in communication is much less than the energy consumed in idle listening. To illustrate this, consider the case where sensor nodes are deployed in a grid topology. Let the node density be 1.96 nodes $/ \mathrm{r}^{*} \mathrm{r}$, on-threshold be 0.7 , and off-threshold be 0.6 . For this setting, the simulation runs for 4541 minutes before the network dies. It turns out that the average number of transmissions per node is 10193 , and the average number of receptions per node is 18202 . Now we use the data from [13] to compute the communication cost on Mica motes. (Similar results can be found for other devices considered in $[17,18]$.) According to [13], the charge required by a Mica mote to transmit a packet is $20 \mathrm{nAh}$, to receive a packet is $8 \mathrm{nAh}$, and to idly listen for 1 millisecond is $1.25 \mathrm{nAh}$. Based on this data, the total communication cost (including transmissions and receptions) in the above simulation is equivalent to the cost that a node stays in idle mode for 4.66 minutes, which is only $0.1 \%$ of the network lifetime (4541 minutes).

\subsection{Global Coverage and Network Lifetime in Networks with Random Topology}

In this section, we study the lifetime-coverage tradeoff in a random topology. We repeat the same set of experiments as in Section 5.1 in a network with random topology. We fix the off-threshold to 0.6 , and vary the on-threshold and node density. The results are shown in Figures 9 and 10.

Based on these results, the performance of pCover under random topology is similar to that where grid topology is used. Moreover, by comparing Figure 8 and Figure 10, we found that, the duration from the point where coverage starts dropping to the point where the network dies is slightly longer in random topology than in grid topology. This is caused by the unevenness of random deployment. In random topology, some areas are covered by more number of sensors, and some areas are covered by fewer number of sensors. The sensor nodes that are deployed in "sparse" areas stay in working mode for a longer period of time than those deployed in "dense" areas. Therefore, the energy consumption is not as well balanced as that in grid topology. However, in random topology, the energy consumption among sensor nodes is still reasonably balanced. For example, when node density is 3.5 nodes/r*r, global coverage is maintained at the desired level (around 94\%) for about 7000 minutes. It then drops to less than $50 \%$ after another 1000 minutes. 


\subsection{If Coverage Area Is Not A Circle}

For simplicity, we assumed that the coverage area of each sensor is a circle. However, pCover can be easily applied to the case where other coverage areas. To illustrate this, we consider a camera sensor. A camera sensor can identify an object within a certain angle of view upto a certain distance. Thus, the coverage area of the camera sensor can be viewed as a sector. We consider the case where the sector is of angle 90 degrees. To keep the area covered by each sensor to be the same as that in case where the coverage area is a circle, we let the sensing range be $20 \mathrm{~m}$ (twice in the case where the coverage area is a circle). In Figure 11, we consider the case where the cameras are deployed in a grid and the angle of each camera is chosen randomly (but fixed throughout the simulation). As we can see from Figure 11(a), varying on-threshold allows us to control the level of coverage and lifetime. Moreover, by comparing Figures 7(b) and 11(b), we can see that the level of coverage and lifetime is comparable to the case where the coverage area is a circle. We perform similar experiments for the case where sensors are deployed randomly (cf. Figure 12). We can see that a similar result also holds in the case where the location of the sensors is randomly chosen as well.

\subsection{Quality of Surveillance}

Degree of coverage, i.e., the percentage of target area that is covered by working sensor nodes, provides one metric to measure the level of network surveillance. In a partially covered sensor network, the quality of surveillance is often measured in terms of how fast the sensors can detect a target object. The target object can be either stationary or moving. Because the sensor nodes wake up every 2 minutes to check the changes in the environment and neighbors' status, the stationary objects can always be detected within 2 minutes. Moreover, since sensor nodes rotate their working load so that different sets of active nodes are selected at different time. The coverage "holes", the areas that are uncovered by any sensor node, change locations continuously. Hence, an uncovered object in a "hole" is likely to be detected at the time nodes change working schedule.

In the case of moving objects, the quality of surveillance is affected by the sizes (or diameters) of the "holes", i.e., the areas that are not covered by any sensor node. We measure the sizes and the diameters of the holes. For comparison, we define the a node's sensing area $\left(\pi r^{2}\right)$ as a unit area, and the diameter of a node's sensing area (2r) as a unit distance. Since we have set the nodes' sensing range $r$ to $10 \mathrm{~m}$, the unit area is $314 \mathrm{~m}^{2}$, and the unit distance is $20 \mathrm{~m}$.

We analyze the quality of surveillance on the case where nodes are deployed in a grid topology, density is 4 nodes/r*r, 
on-threshold and off-threshold are set to 0.7 and 0.6 , respectively. The global coverage is maintained at $94 \%$, and the lifetime of the network is 8824 minutes. We obtain nodes' on/off status periodically. In Figure 13, we show the sizes and diameters of the coverage holes over time. In Figure 14, we show the snapshots of the network at 2500 minutes, 5000 minutes, and 7500 minutes. As shown in Figure 13 (a), the sizes of the holes are small. The maximal size of the holes is close to 0.6 unit area, and the average size of the holes is only 0.05-0.13 unit area. This is reasonable considering the thresholds we are using are 0.7 (on-threshold) and 0.6 (off-threshold), which means a sensor node starts working if it finds that an uncovered area within its sensing range is larger than 0.3 unit area, and it does not turn off if the uncovered area within its sensing range is larger than 0.4 unit area. The holes occasionally become larger when the uncovered areas that are adjacent to each other are connected. However, this happens infrequently.

In Figure 13 (b), we can see that the diameters of the holes are also short. The average diameter of the holes is only 0.26-0.54 unit distance. The maximal diameter of the holes is 2.4 unit distance. This means that if the target object moves fast (before nodes' rescheduling), the maximal distance it can travel before being detected is 2.4 times the diameter of a sensor's sensing area. In a typical surveillance sensor network such as intrusion detection system, the intruder does not know the locations of the holes as they change continuously. It is unlikely that the intruder can choose to move along the longest uncovered path. Hence, the detection latency is expected to be short in practice.

\subsection{Fairness in Energy Consumption}

We have shown in Section 4.1 that $p$ Cover maintains a balanced energy consumption among sensors as all sensors run out of battery at around the same time. In this section, we illustrate the fairness in energy consumption by demonstrating the battery levels of sensors over time. The results are based on the simulation in a network of grid topology. Node density is 4 nodes $/ \mathrm{r}^{*}$ r. On-threshold and off-threshold are set to 0.7 and 0.6 , respectively. The network lifetime in this setting is 8847 minutes. As we mentioned earlier, the initial battery level of an individual sensor is 1000 minutes. In Figure 15, we show the battery levels of sensors in the network after every 2000 minutes. As we can see, the sensors lose their power uniformly.

In Figure 16, we show the average battery level of sensors at different times. We can see that the drop of average sensor battery level is linear over time. Moreover, the battery levels of all the sensors in the network vary within a short range. For example, at time 2000 minutes, the average battery level is 771 minutes, the battery levels of $74 \%$ sensors are in the range between 671 minutes and 871 minutes (average level +/- 100 minutes). And at time 4000 minutes, the average battery level is 542 minutes, $61 \%$ sensors have the battery levels in the range of 442 to 642 minutes. 


\subsection{Changing Level of Coverage Dynamically}

As we saw in Section 4, changing the on-threshold can affect the global coverage and network lifetime. This feature can be used effectively to dynamically change network parameters when requirements change. For example, consider the case where on/off thresholds are chosen based on how long the network is expected to last, e.g., in Figure 17, an on-threshold of 0.7 and off-threshold of 0.6 may be chosen if the network is expected to be used for 8000 minutes. However, if subsequent requirements change so that an additional network lifetime is needed then we can reduce the on threshold and increase the lifetime (at the cost of reduced coverage of course.) To illustrate the ability to change the coverage dynamically in this manner, in Figure 17 (b), we consider the case where on-threshold is changed dynamically after every 2000 minutes. As we can see, when the on/off threshold are changed in this manner, the effective global coverage and lifetime changes accordingly. In Figure 18, we consider the same issue in case of random topology. As we can see from Figure 18 (b), even with random topology, it is possible to dynamically change the coverage and lifetime.

\subsection{Failure of Sensors}

In this section, we consider the performance of $p$ Cover for the case where some sensors fail (immediately) after deployment. We study the effect of such failures in grid topology in random topology. In Figure 19 (grid topology) and 20 (random topology), we consider the case where the number of failed sensor varies from $5 \%$ to $30 \%$. As we can see from these two figures, the reduction in the level of global coverage and the reduction in lifetime are small. Moreover, the reduction is proportional to the number of failed sensors. Also, if we looked at the coverage where $25 \%$ sensors have failed then, the resulting coverage and lifetime is approximately equal to the case where the deployment is uniform with density is 3 nodes/r*r. In other words, the effect of failure is the same as reduction in deployment of sensors. Thus $p$ Cover is able to tolerate failure of sensors gracefully.

\subsection{Limits of Local Protocol for Coverage}

The protocol $p$ Cover is designed for high partial coverage. In this section, we identify limits of $p$ Cover (or similar local protocols) for the case where low level of coverage is desired. Such a situation may occur if a network is deployed in scenarios where the likelihood of event is low and higher detection latencies are acceptable. In Figure 21, we consider the case where $p$ Cover is used with on/off threshold of $\epsilon>0$. In $p$ Cover, each sensor only communicates with nodes that are within distance 
twice the sensing range. For such a network, the lowest level of coverage possible from $p$ Cover is approximately $50 \%$. However, this can be further reduced if the sensor checks the status of nodes that are outside $2 r$, where $r$ is the sensing range. Such communication is not considered in $p$ Cover since these additional nodes do not provide any coverage assistance to the sensor. From Figure 21, we can observe that by increasing communication range, it is possible to decrease the level of coverage to approximately 5\%. In case of random sensors (cf. Figure 22, the added communication range can assist in lowering the global coverage. However, the minimum achievable is more than the corresponding case in the grid topology.

\section{Comparison of High Partial Coverage and Full Coverage}

As we mentioned earlier, although $p$ Cover is designed to provide high partial coverage, it also provides guaranteed $100 \%$ coverage if we set both thresholds to 1 . In this section, we compare the durations that $p$ Cover maintains desired degrees of partial coverage with the duration that it maintains full coverage. In addition, we also compare $p$ Cover with two other node scheduling protocols proposed in [19] and [23], which provide guaranteed full coverage. The simulations were conducted on networks with random topology. Node density varies from 2 nodes $/ r *$ r to 4 nodes $/ r *$ r.

In Figure 23, the left three bars in each density group represent the durations that the protocols proposed in [19], [23], and $p$ Cover maintain full coverage, respectively. The remaining four bars on the right represent the durations that $p$ Cover maintains different degrees of high partial coverage, from $94 \%$ to $84 \%$, in decreasing order from left to right. It shows that by reducing the desired degree of coverage slightly, we can substantially increase (more than double) the duration that the effective coverage is maintained. For example, when the node density is 4 nodes $/ \mathrm{r}^{*}$, the algorithm proposed in [23] is able to maintain full coverage for about 3000 minutes, the global coverage drops below 95\% at around 4000 minutes, and drops below 90\% at around 4500 minutes. The algorithm proposed in [19] is able to maintain full coverage for about 1000 minutes, and the global coverage drops below $90 \%$ at around 1500 minutes. pCover maintains full coverage for a similar amount of time as the algorithm in [23]. If the desired global coverage is $94 \%, p$ Cover can maintain the effective coverage for 7000 minutes, 2.3 times (respectively, 7 times) the duration for which the protocol in [23] (respectively, [19]) maintains full coverage.

Moreover, with the decrement of global coverage, the duration that the coverage is maintained strictly increases. For example, under the same density ( 4 nodes $/ \mathrm{r}^{*} \mathrm{r}$ ), if we reduce the coverage to $91 \%$, the duration that the coverage is maintained at above $91 \%$ is extended to 7850 minutes; if we further reduce the coverage to $87 \%$, the duration that the desired coverage 
is maintained is extended to 9350 minutes. This illustrates the tradeoff between coverage and network lifetime.

We repeat the same experiments for the case where the coverage area is a 90 degree sector. In Figure 24, we compare the durations that $p$ Cover maintains full coverage and different degrees of partial coverage in a grid network (Figure 24 (a)) and in a random topology network (Figure 24 (b)). The same conclusion holds, that is, reducing a little coverage can substantially increase network lifetime. For example, from Figure 24 (a), we can see that in a network with grid topology, pCover maintains $93 \%$ coverage for 4950 minutes, which is 2.1 times the duration that $p$ Cover maintains full coverage.

\section{An Extension to Improve Quality of Surveillance}

We have shown in Section 5.4 that $p$ Cover provides good quality of surveillance due to the fact that the coverage holes are normally small and change locations continuously. Occasionally, the small holes become connected and create a relatively big hole. The holes can be identified only from a "global" view. However, pCover is a "local" protocol. Sensors only have information of their direct neighbors (i.e., the neighbors that are within $2 r$ distance, where $r$ is a sensor's sensing range), and hence, cannot see the coverage holes that spread across multiple sensors' sensing areas. Based on this observation, we extend $p$ Cover so that sensors acquire the position and status information of the neighboring nodes that are outside $2 r$ distance, which we call extended neighborhood. Sensors can utilize this information to determine the coverage holes in a bigger neighborhood, and make sleep/awake decisions accordingly.

To implement this, sensors need to increase their communication range in order to reach the neighbors that are more than one hops away. In our simulation, we set the communication range to be $3 r$ (For Mica2/XSM sensors [3, 4], the communication range is more than 10 times the sensing range). Sensors collect information of the neighbors that are within $3 r$ distance. We modify the sleep eligibility rule so that it is composed of two steps. In the first step, a sensor computes the local coverage of its sensing area (just as what it does in the standard sleep eligibility rule, cf. Section 3.1). If the local coverage is lower than the given on/off threshold, the sensor stays awake. Otherwise, it performs the second step. In the second step, the sensor identifies the coverage holes in its $2 r$ area by taking into account the contributions from all the neighbors that are within $3 r$ distance. If the size of the biggest hole exceeds a certain threshold (which we call maxholethreshold), the sensor stays awake. Otherwise, it goes to sleep. Hence, a sensor goes to sleep only when it meets the two requirements, its local coverage is higher than the on/off threshold, and the sizes of the coverage holes in its $2 r$ area are lower than the maxhole-threshold. We vary the maxhole-threshold from 1 unit area to $1 / 8$ unit area. The simulations were 
done in a grid network with node density 4 nodes $/ \mathrm{r}^{*} \mathrm{r}$. The on/off thresholds are $0.7 / 0.6$, respectively. In Figure 25 , we show the effect of adding this hole size control extension on global coverage and network lifetime. For comparison, we also plot the corresponding simulation results without hole size control. From Figure 25, we can see that when the maxholethreshold is low, sensors maintains a high global coverage, at the price of reduced network lifetime. When we increase the maxhole-threshold to 1 unit area, the global coverage and network lifetime get close to those without hole size control.

We show the sizes and diameters of the coverage holes in the network over time using the extended $p$ Cover when we set the maxhole-threshold to 1/2 unit area and 1/4 unit area in Figures 26 and 27, respectively. We can see that by controlling maximal hole size in sensors' extended neighborhood ( $2 r$ area in this case), we can effectively reduce the sizes and diameters of the holes. On the other hand, reducing hole size is achieved at the cost of reduced network lifetime. Moreover, the maxholethreshold should not be below 1/4 unit area. Because if maxhole-threshold is too low, maintaining a partial coverage has no obvious advantage in terms of extending network lifetime compared to full coverage.

\section{Related Work}

In recent years, several node scheduling approaches $[7,19,21,23,24]$ have been proposed for surveillance sensor networks to minimize energy consumption while maintaining sensing coverage at the desired level. These approaches try to maintain a subset of sensor nodes in working mode and put the remaining nodes to sleep. In [19], a node scheduling scheme is proposed to preserve full sensing coverage by using an off-duty eligibility rule. Based on the rule, a sensor node is allowed to turn off if and only if its neighbor nodes are able to completely cover its sensing area. When a node computes its neighbors' contribution to its sensing coverage, it simplifies the calculation by using "sponsored sectors". Although it guarantees $100 \%$ coverage, it underestimates the area neighbor nodes can cover, and, hence, leads to redundancy and energy waste, as pointed out in [23]. Moreover, to balance energy consumption, the node scheduling operation is divided into rounds, and nodes perform rescheduling at the beginning of each round. This per-round based operation requires global synchronization service.

In [23], each node decides its sleep-awake schedule at the initialization phase. The set of working nodes are able to provide full coverage of the target area at any time. Moreover, a differentiated sensing coverage can be achieved by proportionally extending ( $k$-coverage, $k>1$ ) or shrinking (partial coverage) the work periods. Although this provides a solution on partial coverage as well, the study in [23] was only conducted on 1-coverage or $k$-coverage (where $k>1$ ). Also like [19], the algorithm proposed in [23] requires time synchronization. 
We have compared the performance of the algorithms proposed in [23] and [19] with $p$ Cover in Section 6. We showed that pCover performs similarly as the algorithm in [23] when used to provide full coverage and is able to provide substantially longer network lifetime when used to provide partial (but high) coverage.

Also, the coverage duration in our protocol is close to the theoretical estimate identified in [25]. In particular, if the coverage is $95 \%$ and the node density is 4 nodes $/ \mathrm{r}^{*} \mathrm{r}$, the theoretical upper bound for coverage duration is 9.8 times the lifetime of a single sensor node. In comparison, our protocol maintains $94 \%$ coverage for a duration that is 8.9 times the lifetime of a single sensor node.

In [21], Wang et.al. prove that an area is $k$-covered if all the intersection points in the same area are $k$-covered, where $k \geq 1$. Based on this, they propose the Coverage Configuration Protocol (CCP), in which a sensor node is eligible to turn off if all the intersection points inside its sensing circle are at least $k$-covered. CCP is designed to provide full coverage or higher degrees of coverage. According to the simulation results from [21], the number of active nodes required by CCP to provide 1-coverage in an $\mathrm{r}^{*} \mathrm{r}$ square area is about $0.88-0.92$, which is similar to that required by [23], and is more than twice the number of active nodes required by our protocol, in order to provide $94 \%$ coverage. Therefore, our protocol can provide significantly longer network lifetime (by reducing a little coverage) than CCP.

pCover's probing mechanism is inspired by PEAS [24], which is a density control protocol. In PEAS, a sleeping node wakes up periodically and broadcasts a probing message to its neighbors within a certain range. Any node that is in working mode responds to the probing message by transmitting a reply message. If a probing node receives a reply message before a timeout, it goes back to sleep. Otherwise, it starts working. In PEAS, a working node keeps awake continuously until its physical failure or depletion of power. This is undesirable since it causes unbalanced energy consumption. To address this problem, in [7], the authors propose PECAS as an extension to PEAS. PECAS lets a node go to sleep after it has been working for a given period of time. When a working node sends a probing reply message, it includes the remaining time it will continue working before going to sleep. Thus, the neighbor nodes will wake up in time to take over the sensing area. PEAS and PECAS control the density of working nodes (hence, the degree of coverage) by controlling the probing range. However, the estimate is inaccurate, and, thus, they are unable to provide guarantees on degree of coverage. By contrast, pCover can provide guaranteed full coverage (by setting both thresholds to 1) and the desired level of partial coverage.

Other node scheduling protocols include Randomized Independent Sleeping (RIS) ( [12]) and MESH ( [7]). Since these algorithms do not involve neighbor cooperation, they are not able to self-configure when the environment changes (e.g., node failure, sensing range changes). In a related area, several node scheduling protocols have been proposed for topology control, 
such as LEACH [8], GAF [22] and SPAN [2] for ad hoc networks, and ASCENT [1] for sensor networks. Although they perform in a similar way as to put the redundant nodes to sleep in order to save energy, these topology control protocols only consider communication connectivity, and try to establish a routing backbone. By contrast, the coverage problem addresses the issue of selecting active sensor nodes to cover every physical point in the target area.

The quality of surveillance in a partially covered sensor field has been studied in [7, 14-16,20]. In [7], Gui and Mohapatra define the quality of surveillance metric using the expected traveled distance of a target before it is first detected by any sensor. They propose two node scheduling protocols, PECAS and MESH (as we mentioned earlier), and then evaluate the performance of various sleep planning protocols using the quality of surveillance metric. Unlike the work in [7], we study the tradeoff between full coverage and high partial coverage, and show the increment in network lifetime by reducing a little coverage. And, our protocol can maintain a network lifetime that is close to (about 90\%) of the theoretical upper bound [25]. In [16], Ren et.al. present a mathematical model to analyze the object detection quality under probabilistic coverage. This work is complementary to our work in that the main focus in $[7,14-16,20]$ is to define the quality of partial coverage.

\section{Conclusion and Future Work}

Maintaining a high degree of sensing coverage and prolonging the system lifetime are two conflicting goals for sensor networks. In this paper, we focused on the effect of providing a partial (but high) coverage in sensor networks on network lifetime. We demonstrated that it is possible to obtain a high level of sensing coverage ( $\sim 90 \%)$ while substantially increasing lifetime when compared with protocols that provide a full (100\%) sensing coverage. To illustrate this, we presented a protocol, $p$ Cover, that depends only on local information and does not depend on services such as time synchronization.

We simulated $p$ Cover on networks of grid topology and random topology. The simulation results show that under random topology, our protocol maintains $94 \%$ global coverage for a duration that is 2.3-7 times the duration for which existing protocols ( $[19,23])$ maintain full coverage. Under grid topology, our protocol maintains the same level of coverage for a duration that is $21.4 \%$ longer than that under random topology. Also, as discussed in Section 8, our protocol achieves a lifetime that is about $90 \%$ of the theoretical upper bound on lifetime identified in [25]. Moreover, we show that our protocol provides load balancing. In a network with uniform distribution, all the sensor nodes die within a short period. The desired degree of global coverage is maintained (almost) until the point where all sensor nodes die. We also showed that $p$ Cover performs well in the presence of failed sensors, and it can be used in different sensing coverage areas. 
Based on the simulation results, we found that the relationship between global coverage and on-threshold is fixed, and is independent of node density (as long as the density is high enough to provide the desired coverage). Thus, we can locally control the global coverage by simply tuning the local parameter on-threshold. For a given on-threshold (or global coverage), we can predict the lifetime of the network under a certain node density, or compute the required node density in order to achieve a desired lifetime. We show that network lifetime is proportional to node density, under a certain on-threshold (or global coverage). Our protocol is also useful in dynamic reconfiguration. To illustrate this, consider the scenario when the sensor nodes have been deployed, we find that the network is required to last longer than originally planned. In this case, we can reduce the value of on-threshold in order to reduce the degree of coverage and extend network lifetime. We demonstrated how we achieve a gradually decreasing coverage by controlling the parameter(i.e., on-threshold) dynamically. The new value of on-threshold can also be distributed to all the sensor nodes in the network through a reprogramming service (e.g., [10]).

One of the assumptions we made in this work is that sensors are stationary and each sensor's coverage area does not change. As future work, we plan to investigate the case where sensors' coverage areas change over time. This can be camera sensors that revolve angles constantly or sensors that are attached to robots or vehicles. In this case, we need to decide sensors' moving speed and pattern to achieve the desired degree of global coverage.

\section{References}

[1] A. Cerpa and D. Estrin. ASCENT: Adaptive self-configuring sensor networks topologies. IEEE Transactions on Mobile Computing, 3(3):272-285, 2004.

[2] B. Chen, K. Jamieson, H. Balakrishnan, and R. Morris. Span: An energy-efficient coordination algorithm for topology maintenance in ad hoc wireless networks. In Proceedings of the 6th ACM MOBICOM Conference, July 2001.

[3] Crossbow Technology, Inc. MICA2 Datasheet. http://www.xbow.com/Products/Product_pdf_files/ Wireless_pdf/MICA2_Datasoheet.pdf.

[4] Crossbow Technology, Inc. MSP410 Datasheet. http://www.xbow.com/Products/Product_pdf_files/ Wireless_pdf/MSP 410_Data\%sheet.pdf.

[5] Crossbow Technology, Inc. MSP410, TelosB, MICA2 Datasheets. http://www.xbow.com/Products/ Product_pdf_files/Wireless_pdf/ $\{$ MSP 410, Tel\%osB,MICA2\}_Datasheet.pdf. 
[6] A. Arora et. al. Exscal: Elements of an extreme scale wireless sensor network. The International Conference on Real-Time and Embedded Computing System and Applications (RTCSA), August 2005.

[7] C. Gui and P. Mohapatra. Power conservation and quality of surveillance in target tracking sensor networks. In Proceedings of the Tenth Annual International Conference on Mobile Computing and Networking (ACM MobiCom), October 2004.

[8] W. R. Heinzelman, A. Chandrakasan, and H. Balakrishnan. Energy-efficient communication protocol for wireless microsensor networks. In Proceedings of the 33rd Hawaii International Conference on System Sciences, Janauary 2000.

[9] C. Huang and Y. Tseng. The coverage problem in a wireless sensor network. ACM International Workshop on Wireless Sensor Networks and Applications (WSNA), pages 115-121, 2003.

[10] S. S. Kulkarni and L. Wang. MNP: Multihop network reprogramming service for sensor networks. In Proceedings of the 25th International Conference on Distributed Computing Systems (ICDCS), pages 7-16, June 2005.

[11] S. Kumar, T. H. Lai, and A. Arora. Barrier coverage with wireless sensors. ACM International Conference on Mobile Computing and Networking (MobiCom), 2005.

[12] S. Kumar, T. H. Lai, and J. Balogh. On k-coverage in a mostly sleeping sensor network. In Proceedings of the Tenth Annual International Conference on Mobile Computing and Networking (ACM MobiCom), pages 144-158, October 2004.

[13] A. Mainwaring, J. Polastre, R. Szewczyk, D. Culler, and J. Anderson. Wireless sensor networks for habitat monitoring. In Proceedings of ACM International Workshop on Wireless Sensor Networks and Applications (WSNA'02), Atlanta, GA, September 2002.

[14] S. Meguerdichian, F. Koushanfar, M. Potkonjak, and M. Srivastava. Coverage problems in wireless ad-hoc sensor networks. In Proceedings of IEEE INFOCOM, 3:1380-1387, April 2001.

[15] S. Meguerdichian, F. Koushanfar, G. Qu, and M. Potkonjak. Exposure in wireless ad hoc sensor networks. In Proceedings of 7th ACM International Conference on Mobile Computing and Networking (Mobicom), pages 139-150, July 2001. 
[16] S. Ren, Q. Li, H. Wang, X. Chen, and X. Zhang. Analyzing object detection quality under probabilistic coverage in sensor networks. In Proceedings of the 13th International Workshop on Quality of Service (IWQoS), June 2005.

[17] V. Shnayder, M. Hempstead, B. Chen, G. Allen, and M. Welsh. Simulating the power consumption of large-scale sensor network applications. In Proceedings of ACM International Conference on Embedded Networked Sensor Systems (SenSys), November 2004.

[18] M. Stemm and R. Katz. Measuring and reducing energy consumption of network interfaces in hand-held devices. IEICE Transactions on Communications, E80-B(8):1125-1131, August 1997.

[19] D. Tian and N. D. Georganas. A node scheduling schedule for energy conservation in large wireless sensor networks. Wireless Communications and Mobile Computing Journal, May 2003.

[20] G. Veltri, Q. Huang, G. Qu, and M. Potkonjak. Minimal and maximal exposure path algorithms for wireless embedded sensor networks. In Proceedings of ACM International Conference on Embedded Networked Sensor Systems (SenSys), November 2003.

[21] X. Wang, G. Xing, Y. Zhang, C. Lu, R. Pless, and C. Gill. Integrated coverage and connectivity configuration in wireless sensor networks. In Proceedings of ACM International Conference on Embedded Networked Sensor Systems (SenSys), November 2003.

[22] Y. Xu, J. Heidemann, and D. Estrin. Geography-informed energy conservation for ad hoc routing. In Proceedings of the 6th ACM MOBICOM Conference, July 2001.

[23] T. Yan, T. He, and J A. Stankovic. Differentiated surveillance for sensor networks. In Proceedings of the First International Conference on Embedded Networked Sensor Systems (SenSys), pages 51-62, November 2003.

[24] F. Ye, G. Zhong, J. Cheng, S. W. Lu, and L. X. Zhang. PEAS: A robust energy conserving protocol for long-lived sensor networks. In Proceedings of the 23rd International Conference on Distributed Computing Systems (ICDCS), May 2003.

[25] H. Zhang and J. Hou. On deriving the upper bound of $\alpha$-lifetime for large sensor networks. In Proceedings of the Fifth ACM International Symposium on Mobile Ad Hoc Networking and Computing (MobiHoc), May 2004. 


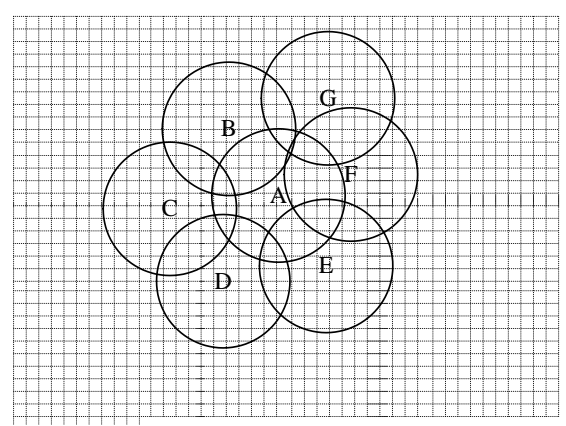

Figure 1. Virtual grid

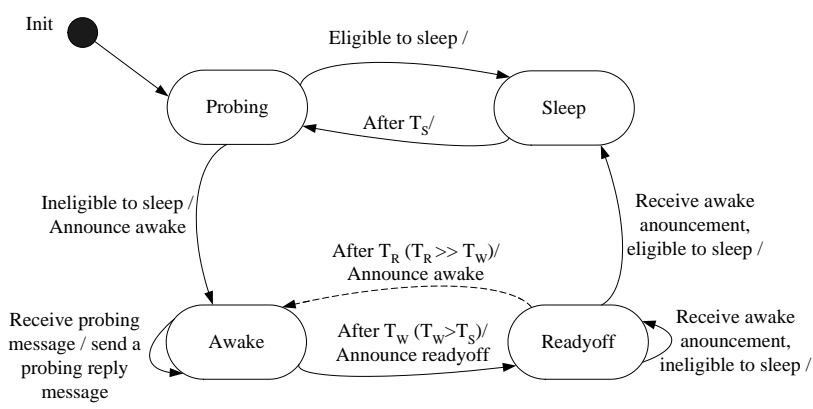

Figure 2. State transition in $p$ Cover

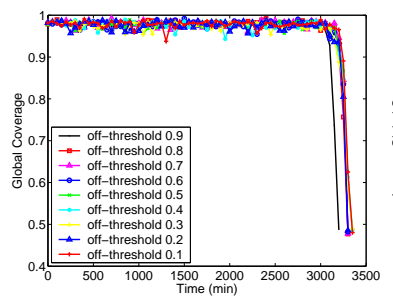

(a)

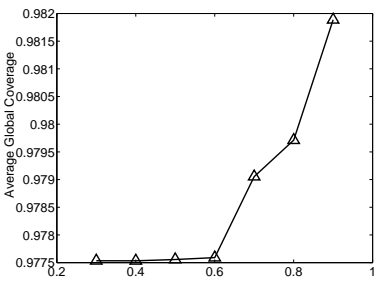

(b)

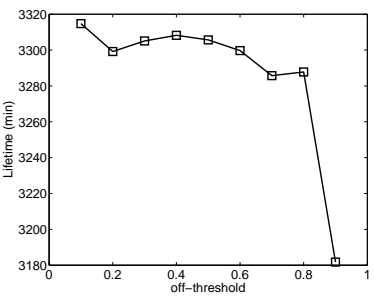

(c)

Figure 3. On-threshold is 0.9 , and off-threshold varies from 0.1 to 0.9 . Node density: 1.96 nodes/r*r. (a) global coverage over time (b) average global coverage vs. off-threshold (c) lifetime of the network vs. off-threshold. 


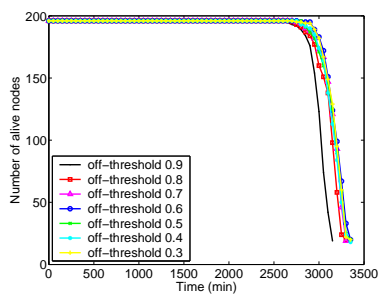

Figure 4. Number of alive nodes over time. on-threshold is 0.9 , and off-threshold varies from 0.1 to 0.9 . Node density: 1.96 nodes/r*r.

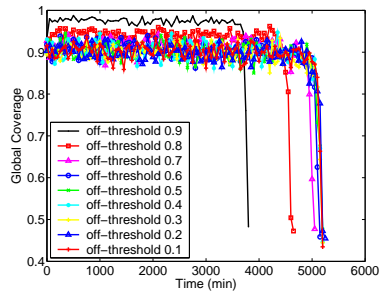

(a)

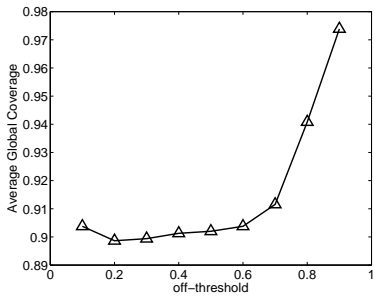

(b)

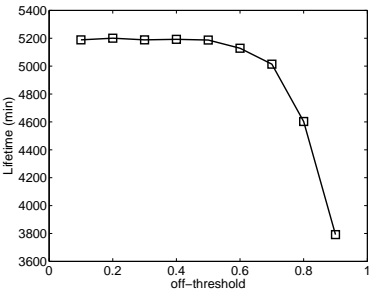

(c)

Figure 5. On-threshold is 0.6 , and off-threshold varies from 0.1 to 0.9 . Node density: 1.96 nodes/r*r. (a) global coverage over time (b) average global coverage vs. off-threshold (c) lifetime of the network vs. off-threshold.

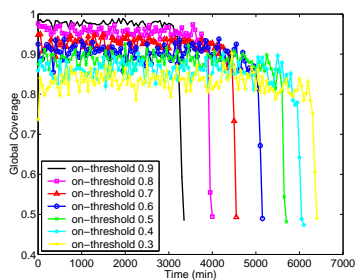

(a)

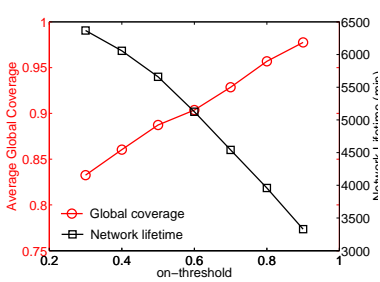

(b)

Figure 6. Off-threshold is 0.6 , and on-threshold varies from 0.3 to 0.9 . Node density: 1.96 nodes/r*r. (a) global coverage over time (b) average global coverage vs. on-threshold and network lifetime vs. on-threshold. 


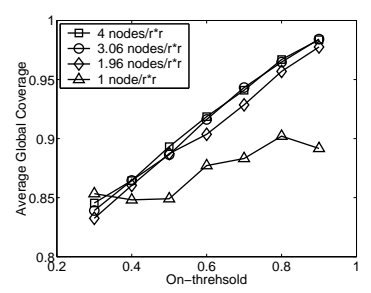

(a)

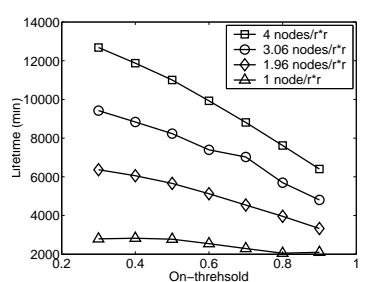

(b)

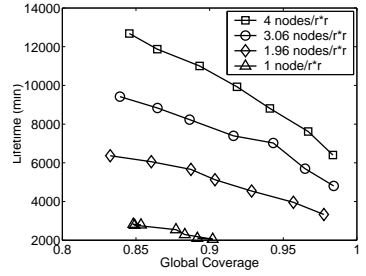

(c)

Figure 7. Grid topology: off-threshold is 0.6. (a) average global coverage vs. on-threshold (b) network lifetime vs. on-threshold (c) network lifetime vs. global coverage.

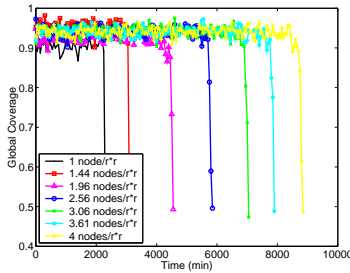

(a)

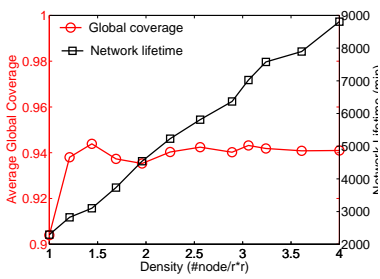

(b)

Figure 8. Grid topology: on-threshold is 0.7 , off-threshold is 0.6. (a) global coverage over time under different node densities (b) average global coverage vs. node density and network lifetime vs. node density.

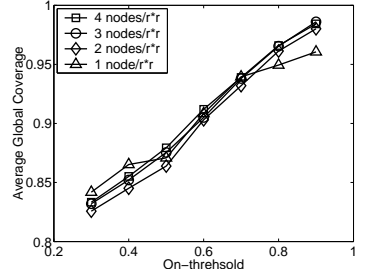

(a)

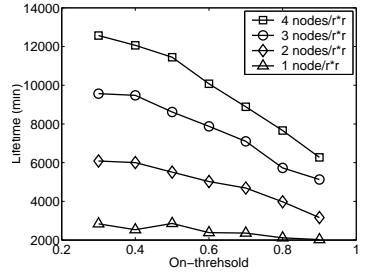

(b)

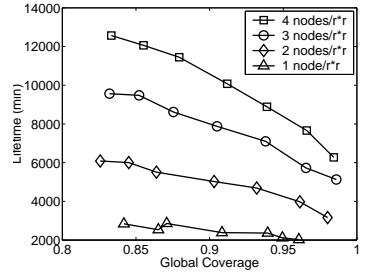

(c)

Figure 9. Random topology: off-threshold is 0.6. (a) average global coverage vs. on-threshold (b) network lifetime vs. onthreshold (c) network lifetime vs. global coverage. 


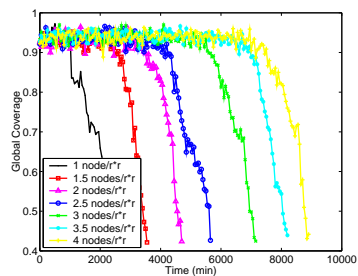

(a)

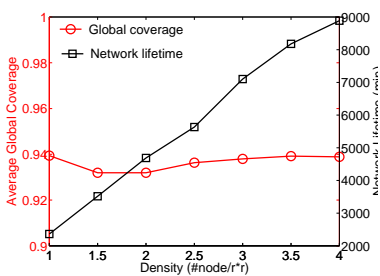

(b)

Figure 10. Random topology: on-threshold is 0.7, off-threshold is 0.6. (a) global coverage over time under different node densities (b) average global coverage vs. node density and network lifetime vs. node density.

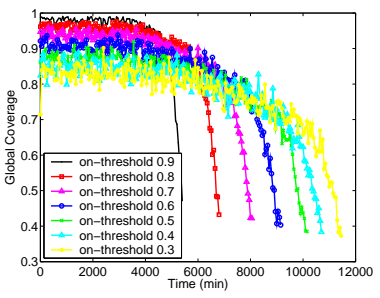

(a)

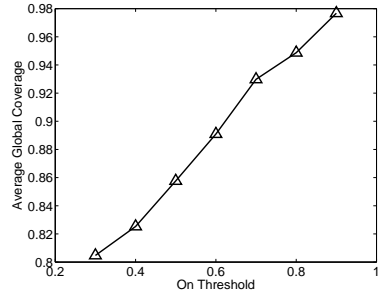

(b)

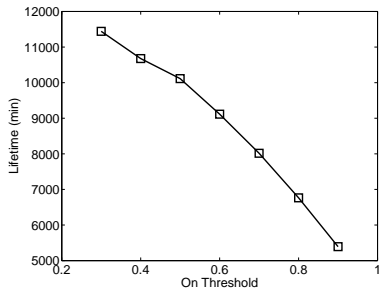

(c)

Figure 11. Sensing range is a 90 degree sector (i.e., $1 / 4$ of a circle). Sensors are deployed in a grid topology. The direction of the sector is randomly chosen. Density is 4 nodes $/ \mathrm{r}^{*}$ r. Off-threshold is 0.6 . On threshold varies from 0.3 to 0.9 . (a) Global coverage over time (b) average global coverage vs. on-threshold (c) network lifetime vs. on-threshold.

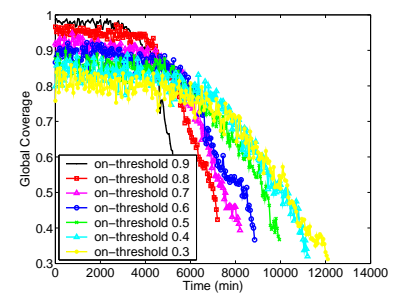

(a)

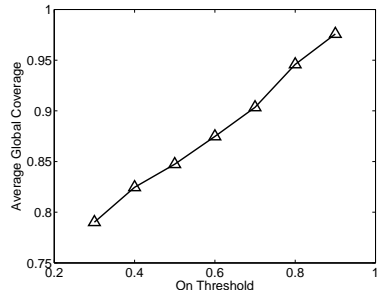

(b)

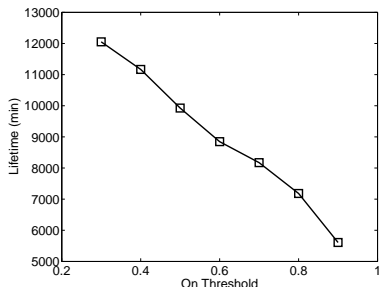

(c)

Figure 12. Sensing range is a 90 degree sector (i.e., $1 / 4$ of a circle). Sensors are deployed in a random topology. The direction of the sector is randomly chosen. Density is 4 nodes/ $\mathrm{r}^{*} \mathrm{r}$. Off-threshold is 0.6 . On threshold varies from 0.3 to 0.9. (a) Global coverage over time (b) average global coverage vs. on-threshold (c) network lifetime vs. on-threshold. 


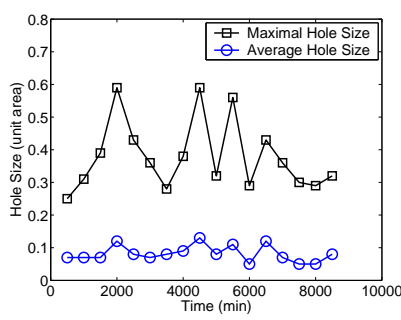

(a)

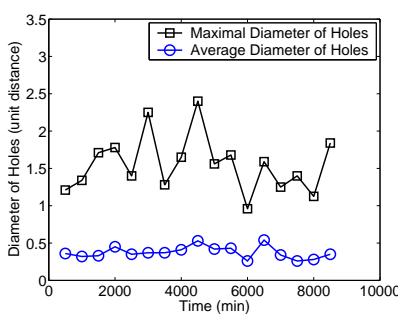

(b)

Figure 13. Sizes and diameters of the coverage holes. on-threshold is 0.7 , off-threshold 0.6 . Node density: 4 nodes/r*r. (a) maximal and average size of the holes (b) maximal and average diameter of the holes. 


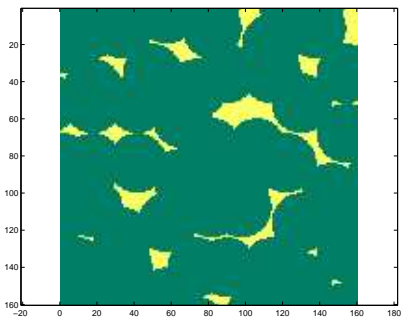

(a)

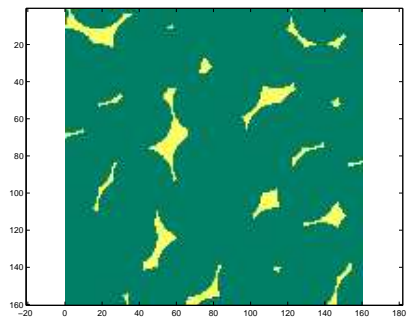

(b)

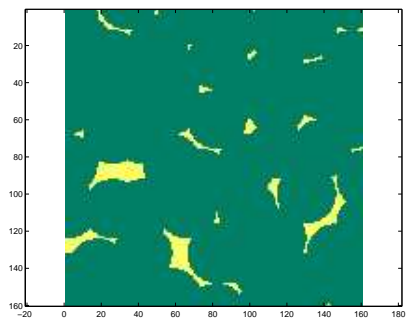

(c)

Figure 14. Snapshots of the network. on-threshold is 0.7 , off-threshold 0.6. Node density: 4 nodes/r*r. (a) 2500min (b)5000min (c) $7500 \mathrm{~min}$.

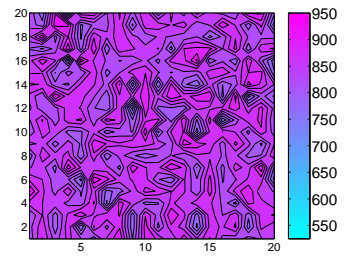

(a)

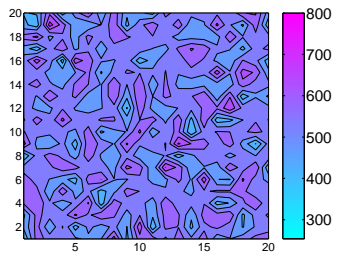

(b)

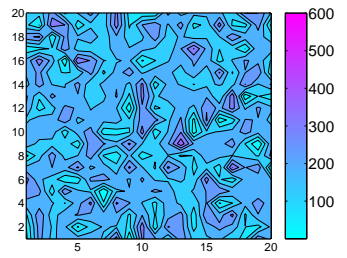

(c)

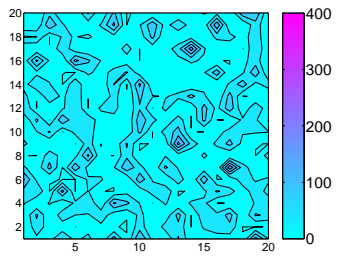

(d)

Figure 15. Battery level of sensors at different times. Grid topology, 4 nodes $/ \mathrm{r}^{*}$, on-threshold is 0.7 , off-threshold is 0.6 . Initial battery level is 1000 minutes. (a) $2000 \min$ (b) $4000 \min$ (c) $6000 \min$ (d) $8000 \mathrm{~min}$. 


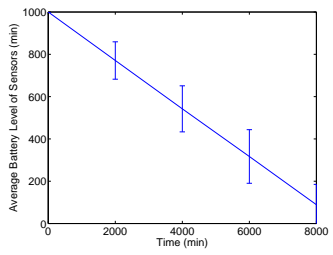

(a)

Figure 16. Average battery level of sensors at different times. Grid topology, 4 nodes/r*r, on-threshold is 0.7 , off-threshold is 0.6 . Initial battery level is 1000 minutes.

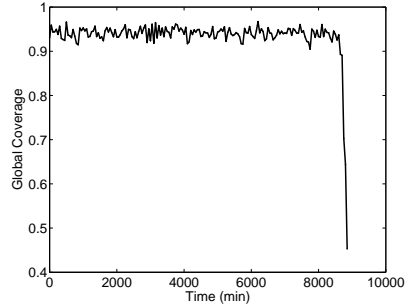

(a)

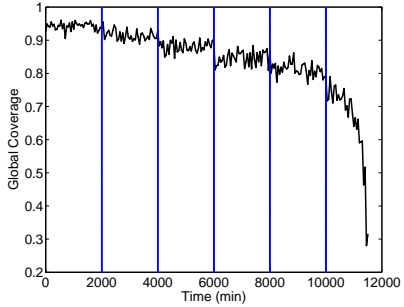

(b)

Figure 17. Gradually reduce level of global coverage. Grid topology. 4 nodes/r*r. Initially on/off thresholds are set to $0.7 / 0.6$. After every 2000 minutes, reduce both on-threshold and off-threshold by 0.1 , until on/off thresholds are $0.2 / 0.1$ (a) Without change of coverage (b) Gradually reduce coverage.

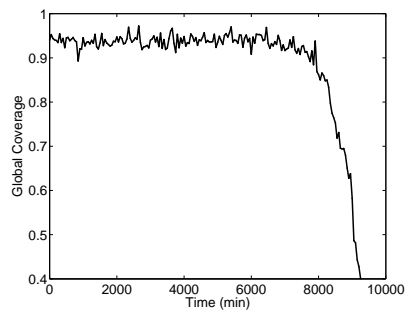

(a)

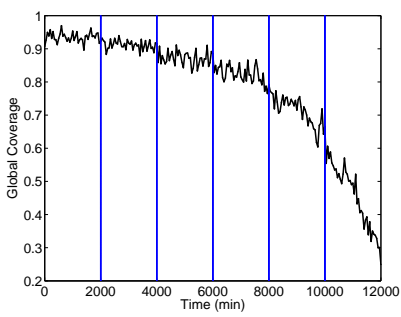

(b)

Figure 18. Gradually reduce level of global coverage. Random topology. 4 nodes/r*r. Initially on/off thresholds are set to $0.7 / 0.6$. After every 2000 minutes, reduce both on-threshold and off-threshold by 0.1 , until on/off thresholds are 0.2/0.1 (a) Without change of coverage (b) Gradually reduce coverage. 


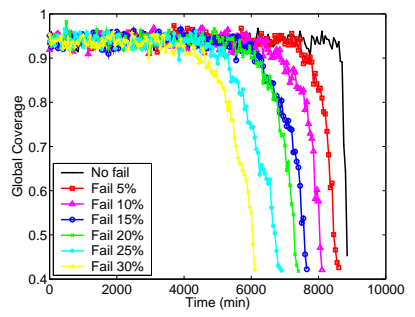

(a)

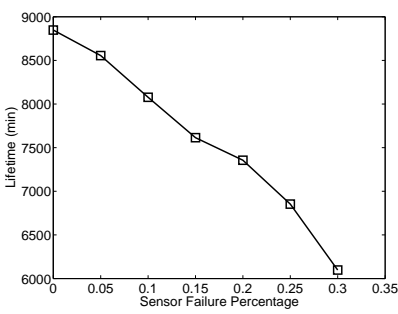

(b)

Figure 19. Randomly fail $5 \%$ to $30 \%$ of sensors in a grid topology. 4 nodes/r*r. On-threshold is 0.7 , off-threshold is 0.6 . (a) Global coverage over time (b) network lifetime vs. sensor failure percentage.

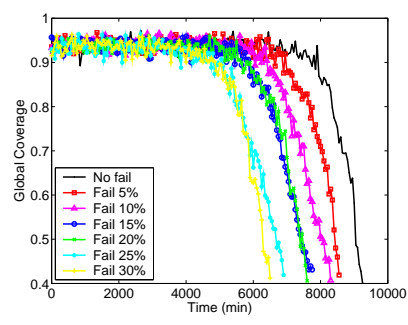

(a)

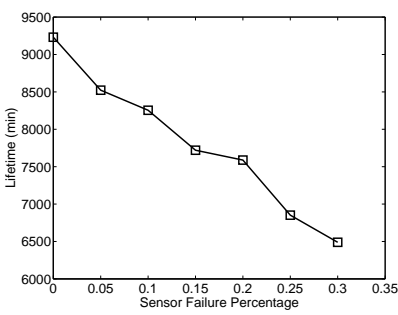

(b)

Figure 20. Fail 5\% to $30 \%$ of sensors in a random topology. 4 nodes/r*r. On-threshold is 0.7 , off-threshold is 0.6 . (a) Global coverage over time (b) network lifetime vs. sensor failure percentage.

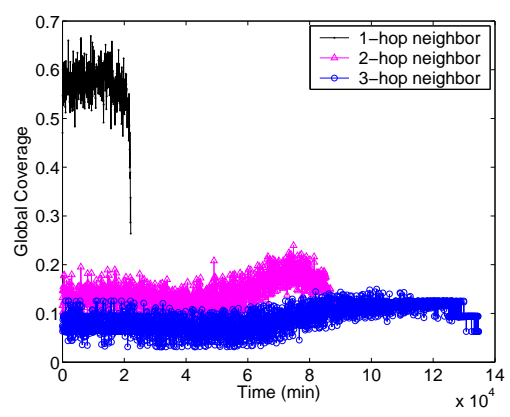

(a)

Figure 21. Lower bound of global coverage. Grid topology. 4 nodes/r*r. 1-hop/2-hop/3-hop neighbors are neighboring sensors within $2 r / 3 r / 4 r$ distance, respectively, where $r$ is the sensing range. 


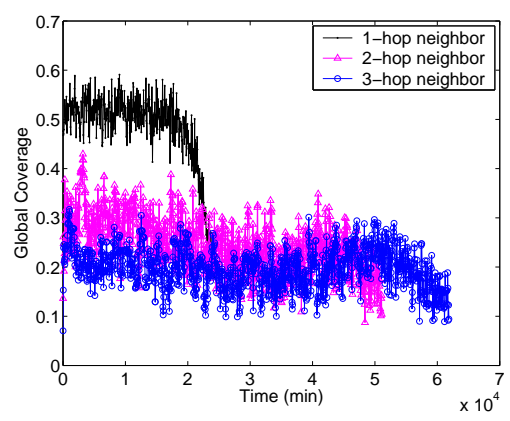

(a)

Figure 22. Lower bound of global coverage. Random topology. 4 nodes/r*r. 1-hop/2-hop/3-hop neighbors are neighboring sensors within $2 r / 3 r / 4 r$ distance, respectively, where $r$ is the sensing range.

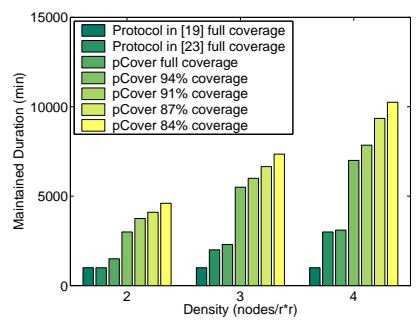

Figure 23. Comparison of high partial coverage and full coverage. Random topology. Sensing range is a circle.

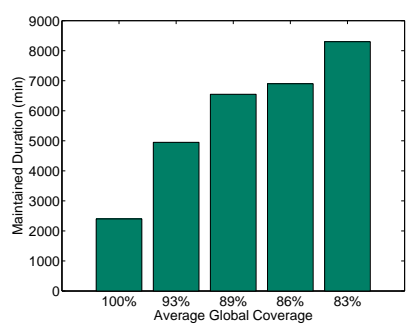

(a)

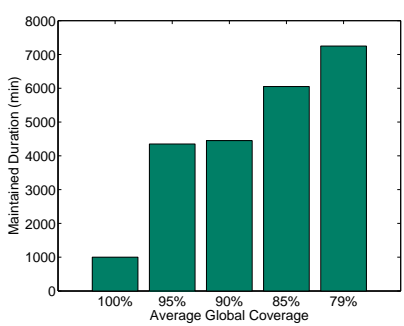

(b)

Figure 24. Comparison of high partial coverage and full coverage when the coverage area is a sector. Grid topology. Sensing range is a sector. 4 nodes $/ \mathrm{r}^{*}$ r. (a) grid topology (b) random topology. 


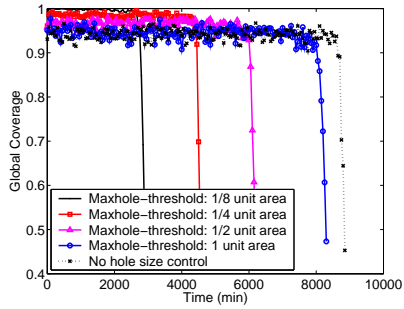

(a)

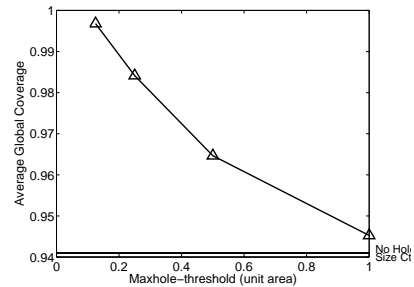

(b)

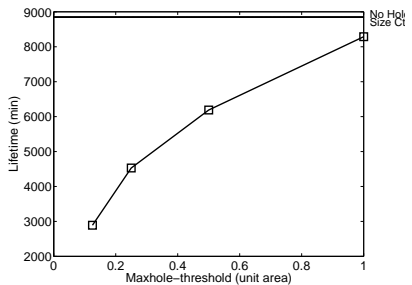

(c)

Figure 25. Extend pCover by adding control of maximal hole size. Grid topology. On-threshold is 0.7, off-threshold 0.6. Node density: 4 nodes/r*r. (a) global coverage over time (b) global coverage vs. maxhole-threshold (c) network lifetime vs. maxholethreshold.

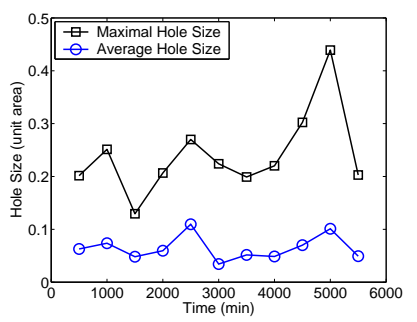

(a)

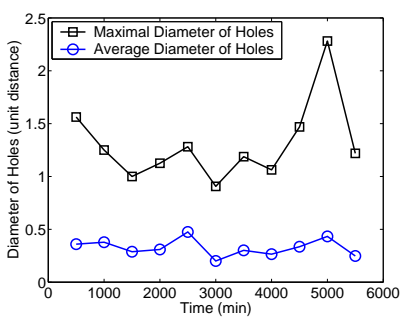

(b)

Figure 26. Sizes and diameters of the coverage holes of extended pCover with maximal hole size control. Grid topology. Onthreshold is 0.7 , off-threshold 0.6. Node density: 4 nodes $/ \mathrm{r} *$ r. Maxhole-threshold is $1 / 2$ unit area. (a) maximal and average size of the holes (b) maximal and average diameter of the holes. 


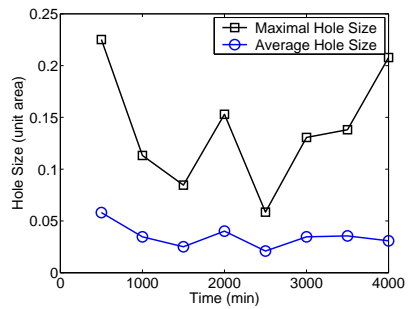

(a)

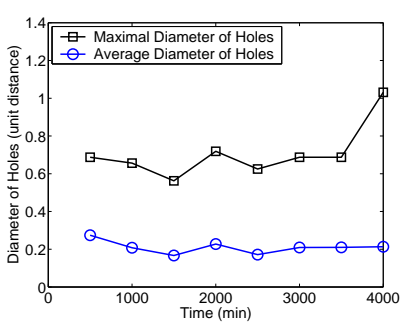

(b)

Figure 27. Sizes and diameters of the coverage holes of extended $p$ Cover with maximal hole size control. Grid topology. Onthreshold is 0.7 , off-threshold 0.6 . Node density: 4 nodes $/ \mathrm{r}^{*} \mathrm{r}$. Maxhole-threshold is $1 / 4$ unit area. (a) maximal and average size of the holes (b) maximal and average diameter of the holes. 